\title{
Investigating the Impact of Changes in Iteration-likelihoods on Design Process Performance
}

Authors: Daniel Shapiro ${ }^{(1)}$ (corresponding author, ds678@cam.ac.uk,+447922058809), Bahram Hamraz $^{(2)}$ (bh351@cam.ac.uk), Anita F. Sommer ${ }^{(1)}$ (afs35@cam.ac.uk),

$$
\text { P. John Clarkson }{ }^{(1)} \text { (pjc10@cam.ac.uk) }
$$

(1) University of Cambridge, Department of Engineering, Engineering Design Centre, Trumpington Street, Cambridge, CB2 1PZ, United Kingdom, www-edc.eng.cam.ac.uk (2) Siemens AG, Corporate Technology, Otto-Hahn-Ring 6, 81739 Munich, Germany

\begin{abstract}
Research on changes in design has mainly focused on the product domain, which is the manifestation of the design process. This article investigates changes in the process domain, which describes the execution of coordinated and concurrent design activities through interdisciplinary teams. More specifically this article focusses on changes in the iterative behavior of activities as one of the key levers determining the performance of complex concurrent design processes. In activity network-based models of design processes the occurrence of such behavior is often expressed probabilistically through iteration-likelihoods.

First, the impact of changes in iteration-likelihoods on the effort and duration of both individual activities and the overall design process is examined through stochastic analysis. Consequently a method for the investigation of changes in iteration-likelihoods is developed, which grounds on an experimental approach using Monte Carlo simulation of activity network-based process models. The method is applied to the design process of a high-speed machining device for the manufacturing of planetary-ball-bearing housings. This analysis results in a two-dimensional, impact-based criticality ranking of potential iteration-likelihood changes and in the identification of the most affected individual activities. The article concludes with managerial implications for process planning and improvement and discusses which design activities need to be targeted by project management to prevent and react to critical iteration-likelihood changes.
\end{abstract}

Key words: product development, design process management, process change, iteration, process simulation. 


\section{Introduction}

Product development (PD) is a key function in industrial organizations and crucial for their success in today's globalized, highly competitive and customer-focused markets. Global competition has put pressure on companies to develop cheaper products of higher quality in less time and to fulfill specific and rapidly changing customer needs, consequently increasing the variety of products drastically $[1,2]$. This has drawn much attention to the management of design processes, which encompass a spectrum of activities at the core of PD and aim at creating recipes for the production of products [3].

The dynamic and complex environment of PD leads to an industrial reality, in which Engineering Changes (EC), loosely defined as changes in released engineering documentation stemming from modifications of the product, are the rule and not the exception [4]. Consequently, since the late 90's manifold methods and tools for Engineering Change Management (ECM) have been developed [5].

However, not only ECs, i.e. changes in the product domain, are likely to occur but also changes in the process domain, e.g., delays in activities, unplanned iterations or new activities added to the process plan. Such process changes can affect major process performance metrics, particularly duration and cost [6-8], and ultimately result in a loss of customer satisfaction. Karniel and Reich [7] acknowledge the relevance of process changes and observe that “... the typical practice has been reactively following changes... rather than proactively planning through analysis of potential changes."

If design research has examined changes in the process domain it has usually focused on activity durations. Common approaches are investigating whether delays of activities lead to process delays, i.e. whether certain activities are on the critical path [9] or assuming probabilistic activity durations and applying simulations to generate process duration histograms [10].

At least since the early 90's also the relevance of iterations for design process performance has been well recognized [11,12]. Understanding iterative behavior is particularly important given the prevalence of the Concurrent Engineering (CE) paradigm in PD practice, as concurrent product realization inherently entails iterative loops [13]. For instance, overlapping dependent activities - a well-established approach within CE - is known as a potential cause of iterations [14]. Also if iterations occur, more effort might be accrued in concurrent than in sequential design processes, as 
larger numbers of activities might be affected by rework due to parallelism. Above all, the major aim of $\mathrm{CE}$ of reducing time-to-market requires efficient management of iterations as these can severely increase design process duration [15].

Most activity network-based process models like DSM [16], GERT [17], Petri nets [18] and IDEF0 [19] include representations of iteration. Such models represent design processes through interconnected discrete activities and are commonly used in engineering design to support visualization, planning, execution and improvement of design processes as they generate understanding of complex process behaviors and associated performance risks [20]. However, activity network-based design process models usually represent iterative behavior only through fixed iterationlikelihoods [21] or iteration-likelihoods that strictly depend on the number of rework cycles [22]. While crucial for process performance of concurrent design processes the impact of uncertainty associated with estimates of iteration-likelihoods is poorly understood.

This article addresses this research gap by investigating the impacts of iteration-likelihood changes on process performance. For this purpose, the design process of a high-speed-machining device for manufacturing of planetary-ball-bearings is first modeled using the Applied Signposting Model (ASM; [23]), an activity network-based process model, and subsequently analyzed through an experimental approach using Monte-Carlo simulations. This analysis results in a two-dimensional, impact-based criticality ranking of potential iteration-likelihood changes as well as an examination of the most affected activities.

The remainder of the paper is organized as follows. Section 2 provides an overview of challenges associated with managing and modeling iterations in design processes. Section 3 first discusses findings on the relationships of iteration-likelihoods, rework-likelihoods, process structure and activity durations, and subsequently defines change impacts on process effort and duration. Then, a simulation-based method for investigating impacts of changes in iteration-likelihood is suggested. This method is applied to the design process of a high-speed-machining device in Section 4 . Section 5 discusses the managerial implications of the results as well as limitations and opportunities for future research. Section 6 summarizes and concludes. 


\section{Iterations in Design Processes}

Design processes are fundamentally about reducing uncertainty in the description of a product. For complex products this is very difficult to realize without any degree of iteration $[11,24,25]$. Although various basic definitions for iteration are proposed (see [26] for an overview of definitions), the term iteration is usually used to describe one of multiple perspectives in design, which can depend on the observer, the design process stage and the product complexity [27]. Six common perspectives on iterations are summarized by Wynn et al. [27]: iterative exploration of design problem and solution, convergence of design parameters upon objectives, refinement of designs, rework because of identified problems or external changes, negotiation of design solutions between designers and repetition of a similar operation on different information.

The first three perspectives regard iteration as something intentional and positive, which ultimately improves the product's quality, while the following two perspectives regard it as something unintentional and rather negative, leading to costly rework of already completed activities [28]. ${ }^{1}$ Such effects of iterations on design process performance, particularly on process duration, cost and product quality, are addressed in manifold studies [15,29]. In extreme cases the combination of interdependent activities with a high level of concurrency, which can decrease process duration but also increase iterations [14,30], might result in very slow or even non-convergent design processes [31].

Therefore it is crucial to plan and sequence design activities so that unnecessary information interdependencies and unintentional iterations are avoided [32-34]. Given that design activities are adequately sequenced, Smith and Eppinger [35] suggest the following measures to decrease the likelihood of unintentional iterations: better coordination or co-location of designers with interdependent work, reduction of team size to increase efficiency, good specification of interfaces and utilization of models to forecast product performance. In addition, the authors [35] name measures to speed up both intentional and unintentional iterations: utilizing CAD systems to accelerate design

\footnotetext{
${ }^{1}$ The last perspective differs from the other perspectives as it describes the repetition of similar operations to achieve different goals, while the others describe the revision of the same goal. The analysis and arguments made in this research rather focus on the first five perspectives.
} 
activities, applying simulations to reduce slow prototype/test cycles, utilizing information systems to accelerate information exchange among designers and eliminating inessential activities.

Although many models include representations of iteration, particularly the modeling of unintentional iteration is very difficult and so far there is no single approach, which is able to comprehensively capture all perspectives on design iteration described above [27,28]. The state of the art in modeling iterations in activity network-based process models is to include cyclic iteration scenarios, which are triggered after the completion of activities and lead to rework of a sub-set of already executed activities. Durations of reworked activities are usually modeled to either correspond to the original activity durations (fixed values or unconditional probability density functions [e.g., 36]) or to fractions of the original activity durations due to learning effects [e.g., 22]. Moreover, the occurrence of iterations is usually modeled in one of three ways. Firstly, Smith's and Eppinger's Work Transformation model [35] and derived publications [6,37] model iterations deterministically, assuming that a number of interdependent and fully parallel activities create a fixed proportion of rework for each other until no work is left. Secondly, the vast majority of models represent iterations probabilistically using fixed iteration-likelihoods as Kusiak and Wang [38] or iteration-likelihoods that decrease with the number of rework cycles to account for learning as Cho and Eppinger [39]. Thirdly, very few models, published by Lévárdy and Browning [40], Suss and Thomson [41] and Wynn et al. [42], represent iterations to occur dependent on the uncertainty or technical risk that is present in the design process.

Furthermore, the few publications that address the sensitivity of the design process to changes in its iterative behavior, e.g., Andersson et al. [43] and Chalupnik et al. [9], do not focus their analysis on this issue but rather discuss it on the side as part of the verification of suggested models. Thus, if discussed at all, only high-level effects on the holistic process duration and effort are examined.

This paper extends this state of the art by analyzing in detail the impacts of changes in iterationlikelihoods on both the holistic design process performance and on individual activities within the process. As will be demonstrated, already small changes in iteration-likelihoods can have a significant 
impact on the effort and duration of concurrent engineering design processes. Nevertheless, this issue is usually not considered in existing activity network-based process models.

To conclude this section some exemplary situations of iteration-likelihood changes are quoted:

1. A design activity, usually executed by an expert designer, is taken over by a novice (iterationlikelihood increase).

2. Tools that are applied in a design activity are enhanced (iteration-likelihood decrease).

3. Requirements of a test activity are increased (iteration-likelihood increase).

4. The original process plan was too optimistic, underestimating iteration-likelihoods.

\section{Measuring Impacts of Changes in Iteration-likelihoods}

This section provides the theoretical background for the investigation of iteration-likelihood changes. First, the relationship between iteration-likelihoods and rework-likelihoods is examined using a simple, hypothetical design process model. Then, based on this relationship the impacts of iterationlikelihood changes on process effort and duration are examined and the possibilities of quantifying these impacts through closed-form analysis versus simulation are assessed. Finally, a simulation-based investigation method for changes in iteration-likelihoods is suggested.

\subsection{Differentiation and Analytical Investigation of Iteration-likelihoods and Rework-likelihoods}

Although an activity's likelihood of iteration contributes to the likelihood that it requires rework, these two likelihoods differ substantially. To clearly distinguish them from each other, they will be referred to as iteration-likelihood and rework-likelihood respectively hereafter.

More precisely, the iteration-likelihood describes the probability that a design activity - often a test or integration activity - does not produce a satisfactory outcome and triggers upstream rework of one or multiple other activities including itself (e.g., the likelihood that a stress analysis fails). An activity's rework-likelihood however describes the probability that the activity is reworked (i.e. executed more than once) within a design process, which can be caused either by its own iteration or by iterations 
triggered by (in)directly dependent activities downstream (e.g., the likelihood that a stress mesh generation activity needs to be reworked because the stress analysis or other activities fail).

This difference between iteration-likelihoods and rework-likelihoods is crucial for the understanding of iteration-likelihood change impacts and thus will be further elaborated based on a simple hypothetical design process model (see Figure 1).
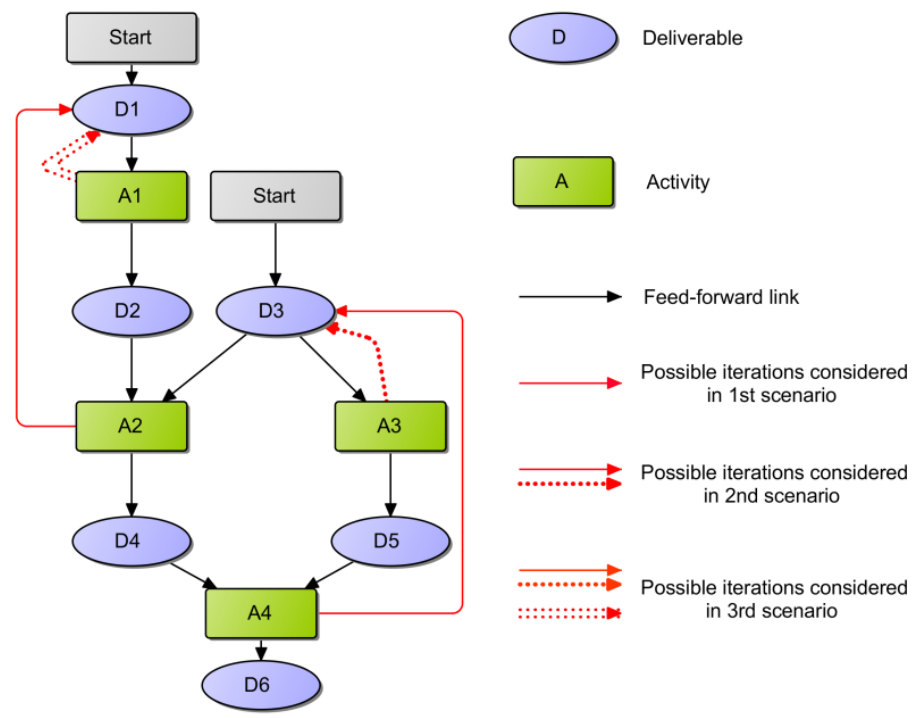

Figure 1: Three different iteration scenarios in a hypothetical design-process model

The model follows the ASM notation [23] and is composed of activities and deliverables interlinked by feed-forward and feedback links. It was intentionally constructed to gain understanding of complex design process behavior, triggered by independent, dependent and interdependent activities [11] that are connected through different logic constructs [7], based on a small number of activities. Iterative behavior is exhibited through several overlapping feedback loops. These loops lead from activities to deliverables to represent that certain inputs might be refined iteratively and that activities need to be reworked following such updates in their inputs.

In the following the iteration-likelihoods $i_{n}, 0 \leq i_{n}<1$, and the durations $d_{n}$ of the activities $n=1, \ldots, N$ are assumed to be independent model inputs, while the rework-likelihoods $r_{n}, 0 \leq r_{n}<1$, are dependent model outputs. 
First, the 1st scenario shown in Figure 1 is analyzed (the dotted iterations are ignored). The reworklikelihoods are derived analytically by determining the respective counter-rework-likelihoods, which allows shorter formal expressions since a counter-rework-likelihood comprises less possible events than a rework-likelihood. For example, the derivation logic for $r_{1}$ is as follows: $A 1$ will not be reworked if $A 2$ does not iterate. However, $A 2$ might be reworked if $A 4$ iterates. Thus, the probability that $A l$ will not be reworked contains the possibility that $A 4$ iterates an arbitrary number of times but the execution of $A 2$ always succeeds. $r_{1}$ can then be expressed applying the sum formula for geometric progressions:

$$
\begin{aligned}
& 1-r_{1}=\left(1-i_{2}\right) \cdot\left(1-i_{4}\right) \sum_{j=0}^{\infty}\left(i_{4} \cdot\left(1-i_{2}\right)\right)^{j}<=> \\
& r_{1}=1-\left(1-i_{2}\right) \cdot\left(1-i_{4}\right) \cdot \frac{1}{1-i_{4} \cdot\left(1-i_{2}\right)} \text {, as } \sum_{j=0}^{\infty}\left(i_{4} \cdot\left(1-i_{2}\right)\right)^{j}<1 .
\end{aligned}
$$

The other rework-likelihoods can be derived applying an analogous logic:

$r_{2}=1-\left(1-i_{2}\right) \cdot\left(1-i_{4}\right)$

$r_{3}=i_{4}$

$r_{4}=i_{4}$

As can be seen, only the rework-likelihood of $A 4$ is independent from other activities. The reworklikelihoods of $A 1, A 2$ and $A 3$ however, depend on the process structure and the iteration-likelihood of at least one other activity in the process. Given these rework-likelihoods, the impact of a change in the iteration-likelihood of activity $m$ on the rework-likelihood of activity $n$ is:

$\Delta r_{n, m}=r_{n}\left(i_{m, n e w}\right)-r_{n}\left(i_{m, o l d}\right)$,

where $i_{m, o l d}$ and $i_{m, n e w}$ are the iteration-likelihoods of activity $m$ before and after the change.

Formulas (1)-(4) also suggest that closed-form expressions can be derived for rework-likelihoods. This would be convenient for investigating the iteration-likelihood change impacts discussed in Section 3.2, as it would allow to develop algorithms for calculating these impacts analytically for a general activity network. However, closed-form expressions cannot be derived for rework-likelihoods for every possible process structure. In fact, examining the 2nd scenario in Figure 1, which includes the 
possibility of self-iterations of $A 3$, demonstrates a fundamental difficulty: the rework-likelihoods of activities do not only depend on iteration-likelihoods but also on durations of other activities. This can be seen when examining $r_{2}$, which could be expressed as follows at first glance:

$1-r_{2}=\left(1-i_{2}\right) \cdot\left(1-i_{3}\right) \cdot\left(1-i_{4}\right)<=>r_{2}=1-\left(1-i_{2}\right) \cdot\left(1-i_{3}\right) \cdot\left(1-i_{4}\right)$

According to Equation (6) $A 2$ is not reworked if $A 2, A 3$ and $A 4$ do not iterate. However, after taking a closer look it becomes clear that an iteration of $A 3$ would not lead to rework in $A 2$ if $A 3$ is completed before $A 2$ has started, because $A 2$ would then be executed for the first time with already updated information from the iteration of $A 3$. This is the case if $d_{3} \leq d_{1}$, assuming that enough resources are available to start both $A 1$ and $A 3$ in parallel at the beginning of the process. Thus, if

1. $d_{3}>d_{1}, r_{2}$ corresponds to the "first-glance-solution" described in Equation (6).

2. $x \cdot d_{3} \leq d_{1}<(x+1) \cdot d_{3}$, where $x$ is a positive natural number (i.e. $d_{3} \leq d_{1}$ ), then

$$
r_{2}=1-\left(\sum_{j=0}^{j=\mathrm{x}} i_{3}{ }^{j}\right) \cdot\left(1-i_{2}\right) \cdot\left(1-i_{3}\right) \cdot\left(1-i_{4}\right)
$$

The second case is more complex: $A 2$ will not be reworked if it does not iterate itself, $A 4$ does not iterate, and $A 3$ does not iterate at all or iterates only up to $x$ times at the beginning of the process, where $x$ depends on the durations of A1 and A3.

In the 3rd scenario in Figure 1 possible self-iterations of $A l$ are added to the example while possible self-iterations of $A 3$ are kept. This makes the calculation of $r_{2}$ even more complicated. For a given pair of durations of $A 1$ and $A 3$, a similar differentiation into two cases as above can be made for each of the theoretically infinite possible numbers of iterations of $A l$ at the beginning of the process.

Therefore, if complex design processes with multiple parallel paths, which contain dozens of interdependent activities, are analyzed, it becomes very difficult to derive rework-likelihoods analytically. This becomes even more challenging if probabilistic activity durations and learning curve impacts are considered [22,44]. Existing research in both mathematics and engineering design $[10,45]$ supports the conclusion that it is not possible at all to express the dynamic behavior of a nonlinear 
system in closed-form. Instead the use of simulations is recommended [21,31]. The following investigation of iteration-likelihood change impacts will thus be based on Monte-Carlo simulations.

\subsection{Impact of Iteration-likelihood Changes on Process Effort and Duration}

The previous section demonstrated how a change in the iteration-likelihood of one activity affects not only its own rework-likelihood but also the rework-likelihoods of other activities in the process. Such rework-likelihood changes directly affect the effort, which is expected to be invested in the respective activities, as rework requires the repeated utilization of resources [10]. Moreover, an increase in rework can also result in a longer process duration [32]. This section analytically investigates the impacts of changes in iteration-likelihoods on process effort and duration, i.e. cost and time, as two major dimensions of design process performance [4].

Hereafter, the effort $c_{n}$ for an activity $n$ will be regarded as the activity's total-execution time $t_{n}$, i.e., the sum of the activity's durations over all rework-cycles, multiplied with the number of designers $\propto_{n}$ required for its execution. Thus, an activity's required effort will be measured in man-days.

Assuming that started activities are always executed with full duration (even if the need for rework is detected) and that neither durations nor the required resources change between rework-cycles, the following relationship exists between the rework-likelihood and the expected effort for an activity $n$ :

$$
\begin{aligned}
& E\left(c_{n}\right)=E\left(\propto_{n} \cdot t_{n}\right)=\propto_{n} \cdot E\left(t_{n}\right)=\propto_{n} \cdot\left[\lim _{x \rightarrow \infty}\left(1-r_{n}\right) \cdot d_{n}+r_{n} \cdot\left(1-r_{n}\right) \cdot 2 d_{n}+r_{n}{ }^{2} \cdot\left(1-r_{n}\right) \cdot\right. \\
& 3 d_{n}+r_{n}{ }^{3} \cdot\left(1-r_{n}\right) \cdot 4 d_{n}+\cdots+r_{n}{ }^{x} \cdot\left(1-r_{n}\right) \cdot(x+1) \cdot d_{n}=\propto_{n} \cdot \frac{d_{n}}{1-r_{n}}
\end{aligned}
$$

where $d_{n}$ is the activity's duration. $E\left(t_{n}\right)$ is intuitively understood as the sum of the total-execution times for 0 to $\infty$ rework-cycles, weighted with the respective probabilities, which depend on the rework-likelihood $r_{n}$. This formulation of an expected effort emphasizes that even without changes in iteration-likelihoods, the effort $c_{n}$ associated with an activity is subject to risk due to the pure possibility of iterations and rework. Because of

$\frac{\partial E\left(c_{n}\right)}{\partial r_{n}}=\propto_{n} \cdot \frac{d_{n}}{\left(1-r_{n}\right)^{2}}>0$,

an activity's expected effort strictly increases with an increase of its rework-likelihood. 
The immediate impact of an iteration-likelihood change of activity $m$ is therefore a change in the rework-likelihood, i.e. $\Delta r_{n, m}$, as well as in the expected effort, i.e. $\Delta E\left(\mathrm{c}_{n, m}\right)=E\left(c_{n, m}\right)-E\left(c_{n}\right)$, of every (in)directly dependent activity $n(n=m$ is possible; see Section 3.1).

As a change in the iteration-likelihood of an activity $m$ usually affects multiple other activities, the resulting change in the expected process effort $E(C)$ equals the sum of the expected effort changes over all activities, i.e. $\Delta E\left(C_{m}\right)=E\left(C_{m}\right)-E(C)=\Delta E\left(\sum_{n=1}^{N} c_{n, m}\right)=\sum_{n=1}^{N} \Delta E\left(c_{n, m}\right)$ for a process with $N$ activities. Limiting this expected process effort is a key responsibility in design process management $[4,10]$. To support comparisons between different processes the corresponding relative measure for changes in the expected process effort will be used hereafter:

$\Delta e\left(C_{m}\right)=\frac{\Delta E\left(C_{m}\right)}{E(C)}=\frac{E\left(C_{m}\right)-E(C)}{E(C)}=\frac{\sum_{n=1}^{N}\left(E\left(c_{n, m}\right)-E\left(c_{n}\right)\right)}{E(C)}$

Changes in iteration-likelihoods do not only affect process effort but also its duration. If activities on the critical path are reworked, the process duration is directly affected. Rework of activities, which are not on the critical path, however, can be absorbed to a certain extent until the delay is so significant that these activities become part of a new critical path themselves [9]. Whether an activity's rework leads to a process-duration increase therefore depends on the process structure. In comparison, rework will always lead to an increase in process effort. Hence, the impacts of iteration-likelihood changes on process duration are more difficult to describe analytically than respective impacts on process effort.

Contrary to the examinations of expected effort described above, it is not meaningful to examine changes in the expected durations of single activities, assuming that an activity's duration and iteration-likelihood are independent. This assumption is commonly made [39] as a respective relationship is difficult to capture in practice. Thus, changes in expected duration will be investigated with regard to the total process rather than the single activity.

Except for the differences discussed above, changes in the expected duration can be analyzed in full analogy to changes in the expected effort. The relative change in the expected process duration after an iteration-likelihood change of activity $m$ is therefore: 
$\Delta e\left(D_{m}\right)=\frac{\Delta E\left(D_{m}\right)}{E(D)}=\frac{E\left(D_{m}\right)-E(D)}{E(D)}$

where $E(D)$ and $E\left(D_{m}\right)$ denote the expected process duration before and after change. Usually both $\Delta e\left(C_{m}\right)$ and $\Delta e\left(D_{m}\right)$ take negative values after a decrease in activity $m$ 's iteration-likelihood and positive values after a respective increase. However, due to complex interrelations between process structure and resource constraints counter-intuitive impacts are possible, where the expected duration decreases when an iteration-likelihood is increased ([9]; although the authors illustrate this counterintuitive behavior for activity delays, the same logic can be applied to activity rework).

Section 3.1 demonstrated that it is not feasible to derive closed-form expressions for $r_{n}$ in complex design processes. As $E\left(c_{n}\right)$ and consequently also $\Delta e\left(C_{m}\right)$ depend on $r_{n}$ for $n=1, \ldots, N$ (see Equations (8) and (10)), it is also not possible to derive $\Delta e\left(C_{m}\right)$ analytically. However, analogous to the rework-likelihoods an estimate for $\Delta e\left(C_{m}\right)$ can be extracted from Monte-Carlo simulations. This requires estimating $E\left(c_{n}\right)$ and $E\left(c_{n, m}\right)$ as the average effort over all simulation runs for every activity $n$ before and after an iteration-likelihood change of activity $m$, which is simultaneously relaxing the stated assumptions for Equation (8). Moreover, it was argued above that $\Delta e\left(D_{m}\right)$ is even harder to derive analytically than $\Delta e\left(C_{m}\right)$. Thus, also $\Delta e\left(D_{m}\right)$ will be extracted from simulations, which requires estimating $E(D)$ and $E\left(D_{m}\right)$ as the average process duration over all simulation runs before and after an iteration-likelihood change of activity $m$. Building upon these findings, a simulationbased method to assess the criticality of iteration-likelihood changes is presented in Section 3.3.

\subsection{Suggested Investigation Method for Iteration-likelihood Changes}

Building upon the theoretical understanding established in Sections 3.1 and 3.2, the following simulation-based method was developed to assess the criticality of iteration-likelihood changes:

1. Run a first set of Monte-Carlo simulations on an activity network-based model of the design process of interest with the originally planned iteration-likelihoods.

2. Obtain estimates for the 1) expected total-execution time $E\left(t_{n}\right)$ and for 2) the expected effort $E\left(c_{n}\right)$ for each activity $n=1, \ldots, N$ as well as for 3) the expected process effort $E(C)$ and for 4) the expected process duration $E(D)$. This requires calculating 1) the average total-execution 
time and 2) the average effort for each activity as well as 3) the average process effort and 4) the average process duration over all simulations respectively.

3. Increase (or decrease) the iteration-likelihood $i_{m}$ of one activity by a fixed number of percentage-points, while keeping the iteration-likelihoods of other activities unchanged and run a set of Monte-Carlo simulations of the adapted design process. Repeat this for each activity $m=1, \ldots, M$, which could be affected by an iteration-likelihood change, so that $M$ sets of Monte-Carlo simulations are run overall.

4. For each of the $M$ simulation sets repeat step 2 thus, resulting in estimates for 1) $E\left(t_{n, m}\right)$, 2) $E\left(c_{n, m}\right)$, 3) $E\left(C_{m}\right)$ and 4) $E\left(D_{m}\right)$ with $m=1, \ldots, M$.

5. For each of the $M$ simulation sets calculate estimates for changes in 1) the expected totalexecution time and 2) the expected effort per activity as well as relative changes in the 3) expected process effort and 4) the expected process duration, i.e. 1) $\Delta E\left(t_{n, m}\right)$, 2) $\Delta E\left(c_{n, m}\right)$, 3) $\Delta e\left(C_{m}\right)$ and 4) $\Delta e\left(D_{m}\right)$ for $m=1, \ldots, M$.

The suggested method is effectively a sensitivity analysis of the expected process effort and duration. Thus, the highest absolute values of $\Delta e\left(C_{m}\right)$ and $\Delta e\left(D_{m}\right)$ indicate the activities with the most critical iteration-likelihood changes. As discussed in Section 5.1, project management might need to trade-off these two process performance dimensions when assessing the criticality of changes.

While $\Delta e\left(C_{m}\right)$ and $\Delta e\left(D_{m}\right)$ can inform project management about the changes with the greatest impacts on the project, $\Delta E\left(t_{n, m}\right)$ and $\Delta E\left(c_{n, m}\right)$ can be used to identify the activities, which are affected the most by such changes. The following section will demonstrate an application of this method based on a case study of the design process of a high-speed machining device.

\section{Application of the Suggested Method to a Case Study}

This section applies the investigation method of iteration-likelihood changes, described in the previous section, to the design process of a high-speed-machining device.

The device was designed at the Technische Universität München under the order of the company Leistritz AG to cut the inner grooves of planetary-ball-bearing housings. High-speed machining implies high loads on fast moving parts and thus, poses strict requirements on both toughness and thermal resistance. Additionally, narrow tolerances and limited access to the bore made this design 
challenging. This design process is well suited as a case study of changes in iteration-likelihoods as it exhibits a highly iterative behavior, which is typical for concurrent design processes, while being of limited scope, so that it allows a comprehensive analysis and discussion at the same time.

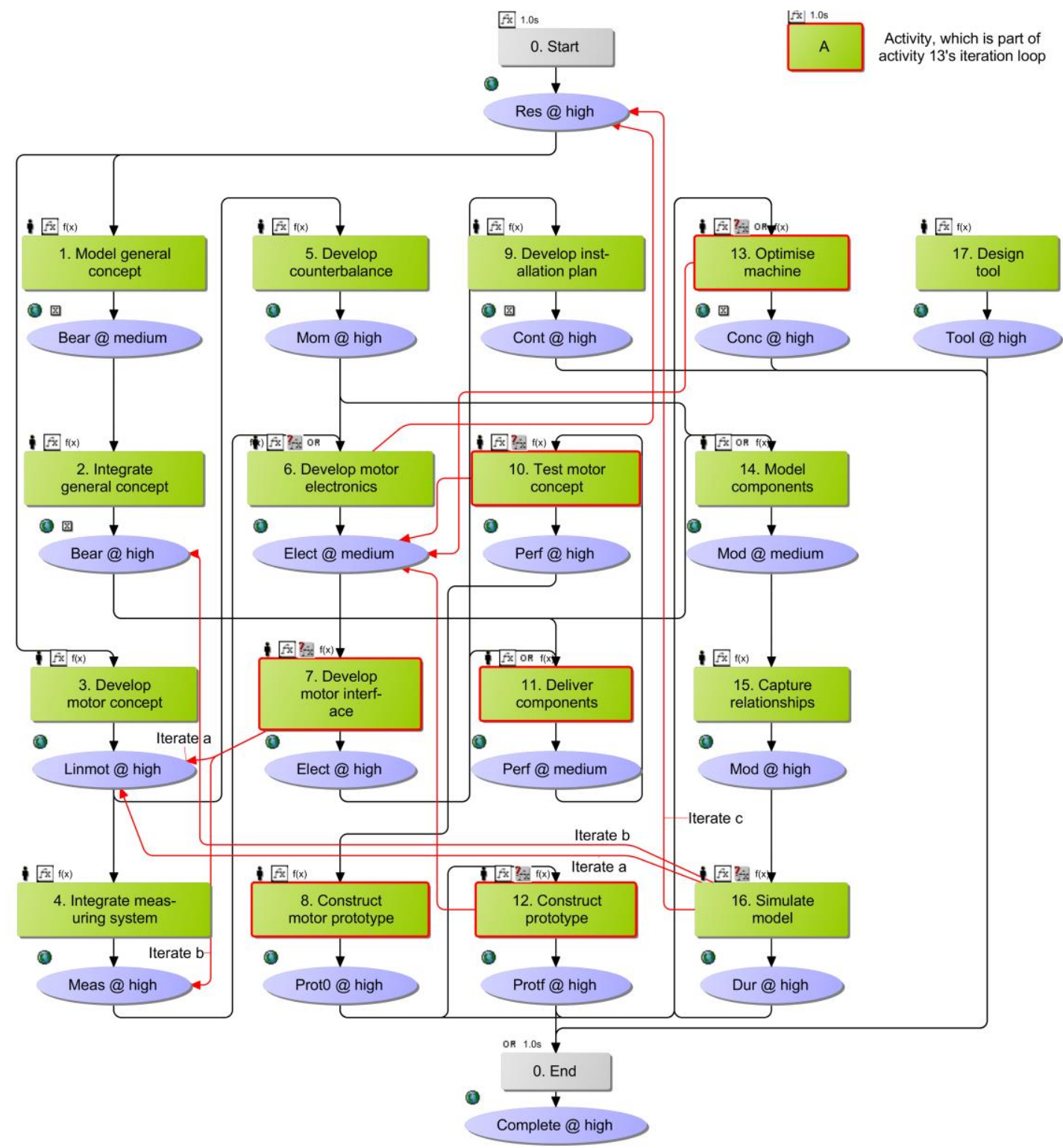

Figure 2: ASM model of the high-speed-machining device design process

Melo [46] built a Signposting model of the high-speed-machining device's design based on individual interviews that he conducted with two designers and the team leader half way through the design process. The estimates of activity durations were extracted from the project's original timetable, while the iteration-likelihoods were estimated by the team-leader based on his experience [46]. Generally, it is difficult to assess the accuracy of iteration-likelihood estimates even after process completion. 
These estimates can be refined over time if multiple similar design processes are executed and the occurrence of iterations is tracked. Also, although not capable of determining the accuracy of iterationlikelihood estimates, the suggested investigation method is useful in this context as it helps understanding the effects of potential errors or changes in such estimates.

For the following analysis Melo's Signposting model was transformed ${ }^{2}$ into an ASM model (see Figure 2) and simulated. ASM is based on a discrete event simulation algorithm, which allows activities to begin if their inputs are either made "available" through the completion of upstream activities or "updated" through iterations. Details on the ASM algorithm can be found in [23].

The process model contains 17 activities, 14 deliverables and nine iteration loops. ${ }^{3}$ It assumes deterministic activity durations and concurrent execution of all independent activities ${ }^{4}$. Simulations of the model result in a mean duration of 245 days and a mean effort of 384 man-days of which 77 mandays are consumed by rework.

The presented analysis is conducted using ten sets of Monte-Carlo simulations: The first set was run with the original iteration-likelihoods. In each of the other sets a single iteration-likelihood was increased by ten percentage-points while the other iteration-likelihoods were kept unchanged. Each set was simulated with 100,000 runs, accumulating to $1,000,000$ process simulation runs altogether, which took approximately 15 hours on a $2.9 \mathrm{GHZ}$ desktop computer. Hereafter, the impacts of iteration-likelihood changes on the expected process effort and duration are discussed first. Then, it is investigated how individual activities are affected by these changes.

The aggregated impacts of the iteration-likelihood changes on the expected process effort and duration are summarized in Figure 3. It shows that a change in activity 13's iteration-likelihood is the most critical with regard to the duration, increasing the expected process duration by $11 \%$, while a change in the likelihood of one of activity 16's iteration loops is the most critical with regard to the effort

\footnotetext{
2 The transformation includes minor modifications accounting for the feedback, which Melo received on the Signposting model from the responsible project manager [43].

3 The underlying ASM process model can be provided upon request. Kindly email the first author. The simulation software CAM is free for research, teaching and evaluation and available for download on https://www-edc.eng.cam.ac.uk/cam.

4 Assuming concurrent execution of independent activities neglects potential resource limitations in the actual project. However it is a useful assumption to emphasize differences in impacts on process effort and duration, as it increases these differences.
} 
(three iteration loops can be triggered through activity $16,16 \mathrm{c}$ being the most critical), increasing the expected process effort by $14 \%$. These impacts are quite severe given that a single iteration-likelihood can easily increase (or even be underestimated) by ten percentage points. On the other hand, e.g., the change in the iteration-likelihood of activity 10 is significantly less impactful with increases of both the expected process effort and duration below $4 \%$. This is because the iteration loop of activity 10 contains less and shorter activities than the iteration loops of activities 13 and $16 \mathrm{c}$.

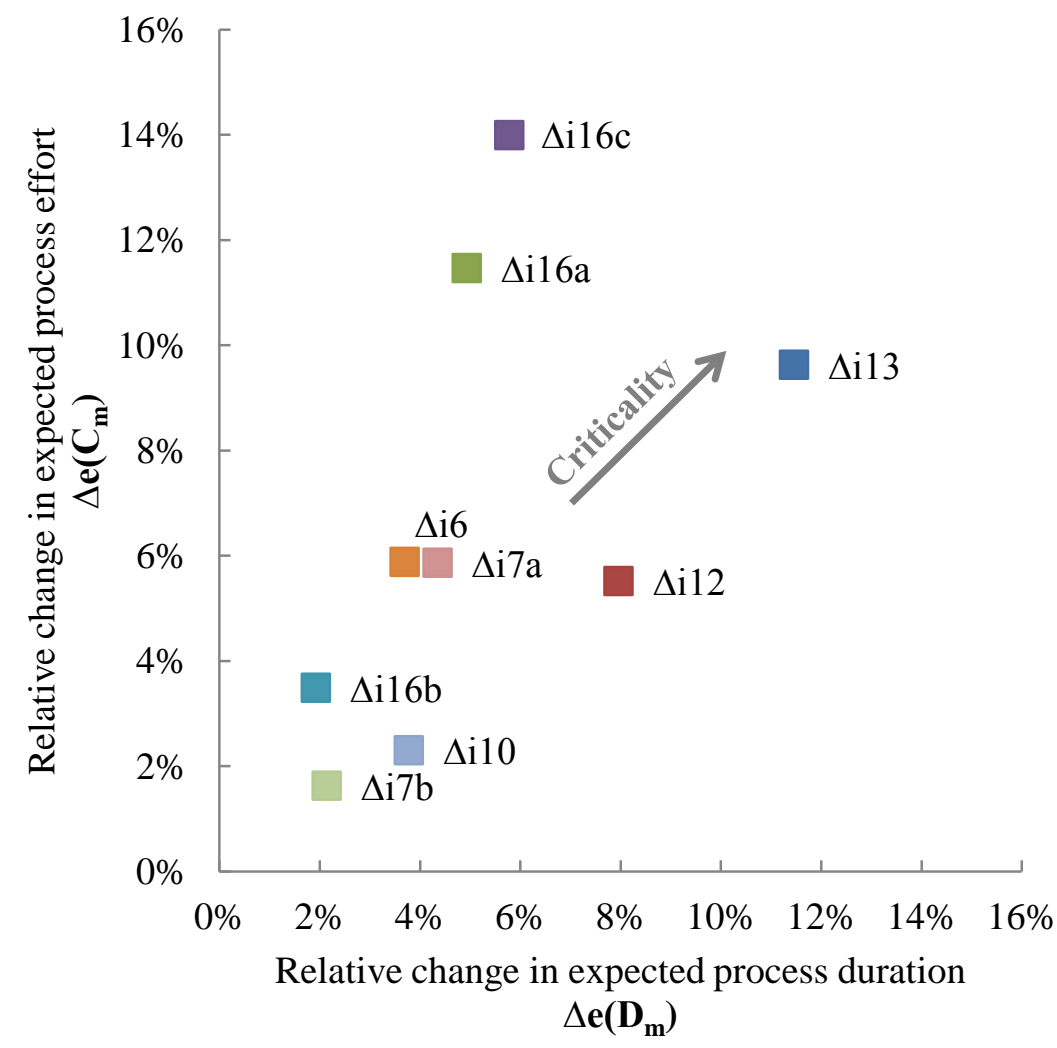

Figure 3: Impacts of increases in iteration-likelihoods by ten percentage-points on expected process effort and duration

In analogy to the Change Prediction Method by Clarkson et al. [47], which is a well-established method for change analysis in the product domain, the investigation method for iteration-likelihood changes does not only identify the most critical changes, but also examines which activities are the most affected by change. Table 1 therefore summarizes for each activity the resulting absolute changes in the expected total-execution time and the expected effort as well as the respective average values for iteration-likelihood increases in different individual activities. It is noteworthy, that the magnitudes of these two measures are only equal in the discussed example, because in the underlying process 
model it is assumed that every activity is executed by a single resource, so that for each activity the total-execution time in days corresponds to its effort in man-days. However, this does not have to be true in the general case. To increase readability the table is colored like a heat map, where green and red cells represent small and large changes respectively.

Table 1: Impacts of increases in iteration-likelihoods by ten percentage-points on expected effort and total-execution time per activity

\begin{tabular}{|c|c|c|c|c|c|c|c|c|c|c|}
\hline \multirow{2}{*}{$\begin{array}{l}\text { Affected } \\
\text { activity } \\
\boldsymbol{n}\end{array}$} & \multicolumn{9}{|c|}{$\begin{array}{l}\text { Change in expected effort/total-execution time per } \\
\text { activity } \Delta E\left(c_{n, m}\right) / \Delta E\left(t_{n, m}\right), \text { man-days/days }\end{array}$} & \multirow[t]{2}{*}{$\varnothing$} \\
\hline & $\Delta \boldsymbol{i}_{\mathbf{6}}$ & $\Delta \boldsymbol{i}_{7 a}$ & $\Delta \boldsymbol{i}_{7 b}$ & $\Delta \boldsymbol{i}_{10}$ & $\Delta i_{12}$ & $\Delta i_{13}$ & $\Delta i_{16 a}$ & $\Delta \boldsymbol{i}_{16 b}$ & $\Delta i_{16 c}$ & \\
\hline 1 & 2 & 0 & 0 & 0 & 0 & 0 & 0 & 0 & 2 & 0 \\
\hline 2 & 0 & 0 & 0 & 0 & 0 & 0 & 0 & 0 & 1 & $\mathbf{0}$ \\
\hline 3 & 1 & 0 & 0 & 0 & 0 & 0 & 0 & 0 & 1 & $\overline{0}$ \\
\hline 4 & 4 & 5 & 0 & 0 & 0 & 0 & 5 & 1 & 6 & 2 \\
\hline 5 & 4 & 5 & 0 & 0 & 0 & 0 & 5 & 1 & 6 & 2 \\
\hline 6 & 2 & 2 & 2 & 0 & 0 & 0 & 2 & 0 & 2 & 1 \\
\hline 7 & 0 & 2 & 2 & 2 & 2 & 2 & 2 & 0 & 2 & 1 \\
\hline 8 & 0 & 0 & 0 & 0 & 1 & 2 & 1 & 0 & 1 & 1 \\
\hline 9 & 0 & 0 & 0 & 1 & 1 & 2 & 2 & 0 & 2 & 1 \\
\hline 10 & 0 & 0 & 0 & 1 & 1 & 1 & 1 & 0 & 1 & 1 \\
\hline 11 & 1 & 0 & 0 & 5 & 6 & 7 & 7 & 3 & 11 & 5 \\
\hline 12 & 0 & 1 & 0 & 0 & 8 & 9 & 8 & 1 & 5 & 3 \\
\hline 13 & 0 & 1 & 0 & 0 & 0 & 12 & 5 & 1 & 3 & 3 \\
\hline 14 & 3 & 2 & 0 & 0 & 0 & 0 & 2 & 2 & 4 & 2 \\
\hline 15 & 1 & 1 & 0 & 0 & 0 & 0 & 1 & 1 & 2 & 1 \\
\hline 16 & 3 & 3 & 0 & 0 & 0 & 0 & 3 & 3 & 6 & 2 \\
\hline 17 & 0 & 0 & 0 & 0 & 0 & 0 & 0 & 0 & 0 & 0 \\
\hline $\begin{array}{l}\text { Total } \\
\text { process }\end{array}$ & 23 & 23 & 6 & 9 & 21 & 37 & 44 & 13 & 54 & 26 \\
\hline
\end{tabular}

Table 1 can be interpreted as follows. First, taking the change in activity 13's iteration-likelihood as an example, it demonstrates that multiple activities (i.e. 7-13) are affected by this change. Activity 9, e.g., would experience and increase in its expected effort by 2 man-days. However, activities 1 to 6 and 14 to 17 are hardly affected by the change. This is because an iteration of activity 13 only leads to rework of these activities if subsequently first activity 7 and then also activity 6 iterate, which is a very unlikely chain of events. Second, Table 1 shows that activity 11 is by far the most affected activity, where the individual iteration-likelihood changes result in an average expected effort increase of 5 man-days (and a corresponding average total-execution time increase). This has two reasons: Firstly, 
activity 11 is part of the feedback loops of multiple other activities (e.g., 10, 12, 13 and 16). Hence, whenever an iteration is triggered by one of these activities, activity 11 requires rework. Secondly, in case activity 11 is reworked, the rework is associated with a substantial effort because activity 11 is labor-intensive (40 man-days) compared to the other activities in the process.

The results are reasonably accurate. For changes in the expected effort of individual activities the $95 \%$-confidence intervals range from \pm 0.0 to \pm 0.3 man-days around the values declared in Table 1 . For changes in the expected effort of the overall design process the 95\%-confidence intervals are wider, ranging from \pm 1.3 to \pm 1.7 man-days around the bottom row values in Table 1 . Given a total expected process effort of $\sim 384$ man-days these potential errors are rather small. To reduce these errors even further the number of simulation runs can be increased. However, for such Monte-Carlo simulations the errors are of order $n^{-0.5}$. Thus, if the simulation runs are increased by a factor of 100 the errors will only decrease by a factor of 10 .

\section{Discussion}

\subsection{Managerial Implications}

Given the understanding of the impacts of potential or actual iteration-likelihood changes gained through the presented analysis, there are two immediate questions that need to be answered to transfer this understanding into managerial decisions:

1. Which changes should project management try to prevent?

2. How can project management prevent or react to such changes?

Examining the first question, it is obvious that an iteration-likelihood change, which has greater impacts on both expected process effort and duration, is more critical than an iteration-likelihood increase where both impact dimensions are smaller. $\Delta i_{13}$, e.g., is clearly more critical than $\Delta i_{10}$ (see Figure 3). However, if for a certain iteration-likelihood change one impact dimension is higher but the second dimension is lower than for another, as it is the case for $\Delta i_{13}$ and $\Delta i_{16 c}$ (see Figure 3), project management needs to trade-off effort versus duration to assess which of the two changes is more critical - a typical decision in design processes [48]. If project management values both effort and 
duration equally, the criticality of an iteration-likelihood change of activity $m$ could be viewed as the distance of the point representing activity $m$ in Figure 3 from the origin, i.e.

Criticality $_{m}=\sqrt{\Delta e\left(C_{m}\right)^{2}+\Delta e\left(D_{m}\right)^{2}}$.

According to this metric $\Delta i_{16 c}$ would be the most critical change with Criticality $_{16 c}=0.15$. However, for the general case project management has to find an appropriate utility-function or weighting, which reflects the importance of effort versus duration for the specific project.

The answer to the second question is not straightforward. To demonstrate this challenge, it is assumed that project management wants to respond to activity 13's change in iteration-likelihood, which was identified as the most critical change regarding process duration (see Figure 3).

Taking a closer look, activity 13 aims at optimizing the overall machine concept, which integrates the linear motor with other components. If this activity iterates, i.e. if the overall machine concept does not fulfill certain requirements, the linear motor concept and the other components will require rework. More precisely, activities 7, 8, 10, 11, 12, 13 (red framed rectangles in Figure 2) and also activity $9^{5}$ will be repeated. Hence, activity 13 constitutes an integration or test activity ${ }^{6}$, which is leading to a large iteration loop of design activities if it iterates [46]. If an increase in the iterationlikelihood of an integration activity needs to be avoided it will not be sufficient to execute only the activity itself with special care. Instead, all of the activities, which are integrated or tested (i.e. the activities in the iteration loop) must be executed with an appropriate quality. Particularly activities, which are integrating further activities themselves, must be executed with a foresight of the downstream integration (activities 7,10 and 12 in the example). To speed-up such an iteration loop and ultimately to reduce the iteration-likelihood of the integration activity the improvement measures suggested by Smith and Eppinger [35], which are discussed in Section 2, could be applied to the process.

\footnotetext{
5 The output of activity 9 does neither directly nor indirectly affect activity 13 . However, activity 9 requires rework after an iteration of activity 13 because activity 9 directly depends on input from activity 7 , which itself is directly affected by the iteration of activity 13 .

6 Melo [46] refers to such an activity as a test task.
} 
The analysis on the extent to which individual activities are affected by changes in iterationlikelihoods (see Table 1) allows detailing this advice. First, if project management generally intends to hedge the project against potential increases in process effort through iteration-likelihood changes, it could focus on decreasing the effort for the activities which absorb the greatest average increase in expected effort without compromising on quality (i.e. activity 11 in the example, see Table 1). However, it is important that the effort for these activities is not traded-off against longer activity durations if these activities are on the critical path of the project. Instead, inefficiencies should be eliminated and suitable computational support to improve information exchange [49] and to decrease the need for physical prototyping could be offered [35] (see also Section 2). Second, if project management generally intends to hedge the project against potential increases in process duration through changes in iteration-likelihoods, it could focus on decreasing the durations of the activities, which are on the critical path of the project and absorb the greatest average increase in total-execution time (this is also activity 11 in the example, see Table 1). Third, to find an appropriate response to a concrete iteration-likelihood change, project management could analogously analyze the impacts of this concrete change instead of the average values. In the above example of an increase of activity 13's iteration-likelihood, activities 11, 12 and 13 itself are affected the most (see Table 1). These activities are all on the critical path, which is the reason why this iteration-likelihood change has the biggest impact on the expected process duration (see Figure 3). Thus, to decrease the impacts of a change in activity 13's iteration-likelihood, project management could particularly focus on decreasing the duration and effort for activities 11, 12 and 13 .

\subsection{Limitations and Future Research}

As demonstrated, an approach was developed to understand, prevent or appropriately react to impacts of iteration-likelihood changes in complex design processes. As in all model-based approaches certain simplifications were made to limit the cost of data gathering and processing. Three relevant simplifications are addressed in the following and directions for future research are derived.

Firstly, throughout this article it is assumed that the iteration-likelihoods of different activities are independent. However, if this assumption does not hold true for a given design process, it is 
straightforward to adapt the suggested analysis method as long as the dependencies between the iteration-likelihoods are known. Instead of running a set of simulations per change in a single iteration-likelihood, a simulation set should then cover changes in the dependent iteration-likelihoods. Secondly, the described procedure is effectively a sensitivity-analysis of complex non-linear systems, where usually different factors are changed by a certain extent and the impacts on the overall system are observed and compared. However, if the change-extent is varied the relative criticality of different factors may vary as well due to the non-linearity of the system. To account for such dynamics this analysis was repeated for iteration-likelihood changes of $-5,+20$ and +30 percentage-points, but did not result in significant variations in the criticality ranking.

Lastly, the case study demonstrated that the analysis results can help focusing project management's attention on a small number of activities to avoid major process performance decreases through iterations. More detailed managerial guidance would additionally require capturing the design team's knowledge and experience on the relationships between upstream activities and the iterationlikelihoods of downstream test/integration activities. This could include the relationships between downstream iteration-likelihoods and 1) the iteration-likelihoods (which would also address the first limitation at the same time), 2) the durations and 3) the execution quality of upstream activities. Currently, such relationships are not captured in common activity network-based process models. Thus, appropriate model extensions constitute a promising opportunity for future research.

\section{Conclusion}

Research on changes in design has mainly focused on engineering changes, i.e. changes in the product domain, which is the manifestation of the design process. This article takes a different perspective and investigates changes in the process domain, which is characterized by the execution of coordinated and concurrent design activities with complex interdependencies and iterative behavior.

More precisely, changes in the likelihood of iterations are examined, which is a key change type in the process domain as even small changes can have significant impacts on design process performance. Therefore, iteration-likelihood change impacts on the expected effort and duration are investigated for 
individual activities and for the overall process. For this purpose, a simulation-based investigation method is developed, which grounds on a stochastic analysis of the relationships of iterationlikelihoods, rework-likelihoods, process structure, activity durations, expected efforts and durations.

The developed method produces a two-dimensional, impact-based criticality ranking of potential iteration-likelihood changes as well as an overview to what extent individual activities are affected by such changes. Based on these insights the method can support project managers in preventing or appropriately reacting to undesirable changes, directing their attention on a small number of activities.

The following conclusions were drawn in the discussion of managerial implications (see Section 5.1): It is argued that project management should focus on preventing the most critical iteration-likelihood changes, which can be identified through a project-specific weighting of the respective impacts on process effort and duration (see Figure 3). Preventing or appropriately reacting to an iterationlikelihood change of one activity often requires executing all of the activities that it tests or integrates with due diligence (see Figure 2). However, simulation results can indicate activities that could be targeted first: To reduce the change impact on process effort project management could focus on decreasing the effort of the activities, which absorb the greatest increase in expected effort without compromising quality. To reduce the change impact on process duration project management could focus on decreasing the durations of the activities on the critical path, which absorb the greatest increase in their accumulated duration (over all repetitions), without compromising quality.

Results indicate that modeling the occurrence conditions of iterations through fixed iterationlikelihoods, as currently is common practice (see Section 2), might not be sufficient because even small changes in iteration-likelihoods can have significant impacts on process effort and duration in concurrent engineering design processes (see Figure 3). Also, more detailed managerial guidance, beyond recommending effort or duration reductions in some activities, might be generated through understanding and considering the relationships between upstream activities and iteration-likelihoods of activities downstream. Yet, only few publications on activity network-based design process models have investigated such relationships (see Section 2), which thus, seems a promising direction for future research. 


\section{References}

[1] Da Silveira, G., Borenstein, D., and Fogliatto, F. S., 2001, "Mass customization: Literature review and research directions," International Journal of Production Economics, 72(1), pp. 1-13.

[2] Holman, R., Kaas, H.-W., and Keeling, D., 2003, "The future of product development," McKinsey Quarterly, (3), pp. 28-39.

[3] Reinertsen, D., 1999, “Lean thinking isn't so simple,” Electronic Design, 47(10), p. 48.

[4] Clark, K. B., and Fujimoto, T., 1991, Product development performance: Strategy, organization, and management in the world auto industry, Harvard Business School Press, Boston, MA, USA.

[5] Hamraz, B., Caldwell, N. H. M., and Clarkson, P. J., 2013, "A holistic categorisation framework for literature on engineering change management," Systems Engineering, 16(4), pp. 473-505.

[6] Cronemyr, P., Öhrwall Rönnbäck, A., and Eppinger, S. D., 2001, “A decision support tool for predicting the impact of development process improvements," Journal of Engineering Design, 12(3), pp. 177-199.

[7] Karniel, A., and Reich, Y., 2013, "Multi-level modelling and simulation of new product development processes," Journal of Engineering Design, 24(3), pp. 185-210.

[8] Khoo, L. P., Chen, C.-H., and Jiao, L., 2003, "A dynamic fuzzy decision support scheme for concurrent design planning," Concurrent Engineering Research and Applications, 11(4), pp. 279-288.

[9] Chalupnik, M. J., Wynn, D. C., Eckert, C. M., and Clarkson, P. J., 2007, “Understanding design process robustness: A modelling approach," 16th International Conference on Engineering Design, ICED 2007, Paris, France, pp. 455-456.

[10] Browning, T. R., and Eppinger, S. D., 2002, "Modeling impacts of process architecture on cost and schedule risk in product development," IEEE Transactions on Engineering Management, 49(4), pp. 428-442.

[11] Eppinger, S. D., 1991, "Model-based approaches to managing concurrent engineering," Journal of Engineering Design, 2(4), pp. 283-290.

[12] Cooper, K. G., 1993, “The rework cycle: Benchmarks for the project manager,” International Journal of Project Management, 24(1), pp. 17-22.

[13] Prasad, B., 1996, Concurrent engineering fundamentals: Integrated product and process organization, Prentice Hall, Upper Saddle River, NJ, USA.

[14] Smith, R. P., and Eppinger, S. D., 1998, "Deciding between sequential and concurrent tasks in engineering design," Concurrent Engineering Research and Applications, 6(1), pp. 15-25.

[15] Ford, D. N., and Sterman, J. D., 2003, "Overcoming the 90\% syndrome: Iteration management in concurrent development projects," Concurrent Engineering Research and Applications, 11(3), pp. 177-186.

[16] Browning, T. R., 2001, "Applying the design structure matrix to system decomposition and integration problems: a review and new directions," IEEE Transactions on Engineering Management, 48(3), pp. 292-306.

[17] Pritsker, A. A. B., 1966, GERT: Graphical Evaluation and Review Technique, Rand Corporation.

[18] Kusiak, A., and Yang, H.-H., 1993, "Modeling the design process with Petri nets," Concurrent Engineering, H.R. Parsaei, and W.G. Sullivan, eds., Springer US, pp. 447-464.

[19] NIST, 1993, "National Technical Information Service, U.S. Department of Commerce, Federal Information Processing Standards Publication 183 (FIPSPUB 183), Integration definition for function modeling (IDEF0)." 
[20] Browning, T. R., and Ramasesh, R. V., 2007, “A survey of activity network-based process models for managing product development projects," Production and Operations Management, 16(2), pp. 217-240.

[21] Smith, R. P., and Eppinger, S. D., 1997, "A predictive model of sequential iteration in engineering design,” Management Science, 43(8), pp. 1104-1120.

[22] Ahmadi, R., Roemer, T. A., and Wang, R. H., 2001, "Structuring product development processes," European Journal of Operational Research, 130(3), pp. 539-558.

[23] Wynn, D. C., 2007, "Model-based approaches to support process improvement in complex product development," $\mathrm{PhD}$ thesis, University of Cambridge.

[24] Loch, C., Mihm, J., and Huchzermeier, A., 2003, "Concurrent engineering and design oscillations in complex engineering projects," Concurrent Engineering Research and Applications, 11(3), pp. 187-200.

[25] Yassine, A., and Braha, D., 2003, "Complex concurrent engineering and the design structure matrix method," Concurrent Engineering Research and Applications, 11(3), pp. 165-176.

[26] Le, H. N., 2012, "A Transformation-based model integration framework to support iteration management in engineering design," $\mathrm{PhD}$ thesis, University of Cambridge.

[27] Wynn, D. C., Eckert, C. M., and Clarkson, P. J., 2007, "Modelling iteration in engineering design," 16th International Conference on Engineering Design, ICED 2007, Paris, France, pp. 693-694.

[28] Browning, T. R., 1998, "Modeling and analyzing cost, schedule, and performance in complex system product development," $\mathrm{PhD}$ thesis, Massachusetts Institute of Technology.

[29] Le, H. N., Wynn, D. C., and Clarkson, P. J., 2012, "Impacts of concurrency, iteration, design review, and problem complexity on design project lead time and error generation," Concurrent Engineering Research and Applications, 20(1), pp. 55-67.

[30] Yang, Q., Zhang, X., and Yao, T., 2012, “An overlapping-based process model for managing schedule and cost risk in product development," Concurrent Engineering Research and Applications, 20(1), pp. 3-17.

[31] Mihm, J., Loch, C., and Huchzermeier, A., 2003, "Problem-solving oscillations in complex engineering projects," Management Science, 49(6), pp. 733-750.

[32] Huang, E., and Chen, S.-J., 2006, "Estimation of project completion time and factors analysis for concurrent engineering project management: A simulation approach," Concurrent Engineering Research and Applications, 14(4), pp. 329-341.

[33] Zhang, H., Qiu, W., and Zhang, H., 2006, “An approach to measuring coupled tasks strength and sequencing of coupled tasks in new product development," Concurrent Engineering Research and Applications, 14(4), pp. 305-311.

[34] Whitfield, R. I., Duffy, A. H. B., Coates, G., and Hills, W., 2003, "Efficient process optimization," Concurrent Engineering Research and Applications, 11(2), pp. 83-92.

[35] Smith, R. P., and Eppinger, S. D., 1997, "Identifying controlling features of engineering design iteration,” Management Science, 43(3), pp. 276-293.

[36] Belhe, U., and Kusiak, A., 1995, "Resource constrained scheduling of hierarchically structured design activity networks," IEEE Transactions on Engineering Management, 42(2), pp. 150-158.

[37] Wang, Z., and Magee, C. L., 2012, "Convergence analysis and iteration estimation for a coupled design process with overlap in redesign," IEEE Transactions on Engineering Management, 59(4), pp. 621-633.

[38] Kusiak, A., and Wang, J., 1993, "Efficient organizing of design activities," International Journal of Production Research, 31(4), pp. 753-769. 
[39] Cho, S. H., and Eppinger, S. D., 2005, "A simulation-based process model for managing complex design projects," IEEE Transactions on Engineering Management, 52(3), pp. 316-328.

[40] Levardy, V., and Browning, T. R., 2009, “An adaptive process model to support product development project management," IEEE Transactions on Engineering Management, 56(4), pp. 600-620.

[41] Suss, S., and Thomson, V., 2012, "Optimal design processes under uncertainty and reciprocal dependency," Journal of Engineering Design, 23(10-11), pp. 826-848.

[42] Wynn, D. C., Grebici, K., and Clarkson, P. J., 2011, "Modelling the evolution of uncertainty levels during design," International Journal on Interactive Design and Manufacturing, 5(3), pp. 187-202.

[43] Andersson, J., and Pohl, J., 1998, "A design process modeling approach incorporating nonlinear elements," Proceedings of 1998 DETC: ASME Design Theory and Methodology Conference, Atlanta, USA, DETC98-5663.

[44] Kara, S., and Kayis, B., 2005, "The effect of the learning process in concurrent engineering projects," Concurrent Engineering Research and Applications, 13(3), pp. 209-217.

[45] Devaney, R. L., 2003, An introduction to chaotic dynamical systems, Westview Press, Boulder, $\mathrm{CO}, \mathrm{USA}$.

[46] Melo, A. F., 2002, “A state-action model for design process planning," $\mathrm{PhD}$ thesis, University of Cambridge.

[47] Clarkson, P. J., Simons, C., and Eckert, C. M., 2004, "Predicting change propagation in complex design," Journal of Mechanical Design, 126(5), pp. 788-797.

[48] Cohen, M. A., Eliashberg, J., and Ho, T.-H., 1996, "New product development: The performance and time-to-market tradeoff," Management Science, 42(2), pp. 173-186.

[49] Prasad, B., Wang, F., and Deng, J., 1997, "Towards a computer-supported cooperative environment for concurrent engineering," Concurrent Engineering Research and Applications, 5(3), pp. 233-251. 

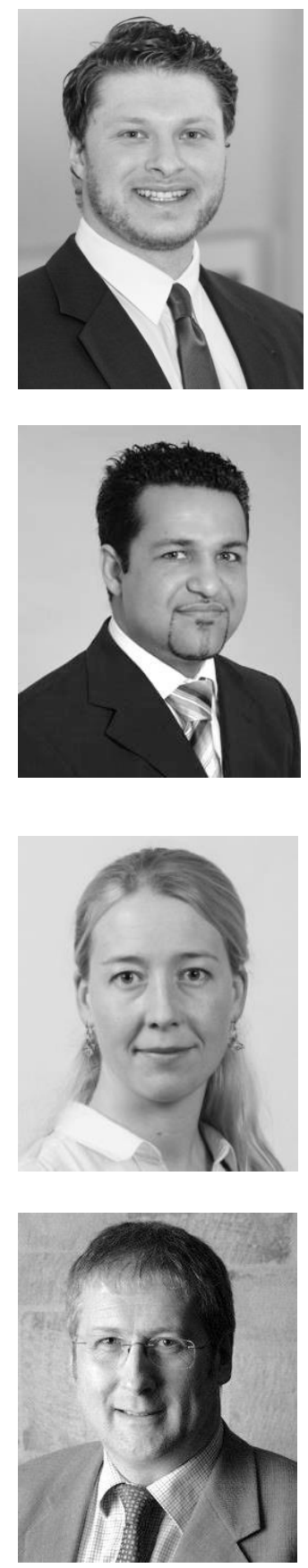

Daniel Shapiro is a PhD Candidate at the Cambridge University Engineering Department. He received a "Diplom-Ingenieur" degree in Industrial Engineering and Management from the Berlin University of Technology in 20011. After two years of working as a management consultant, he joined the Engineering Design Centre at the University of Cambridge in 2013. As member of the Design Processes and Systems group, he investigates changes and their propagation in complex design processes.

Bahram Hamraz is a Senior Consultant at Siemens Corporate Technology. He received his PhD in Engineering Design from the University of Cambridge in 2013 and a "Diplom-Ingenieur" degree in Industrial Engineering and Management from the Hamburg University of Technology in 2007. Bahram's research focuses on engineering change and process management. He is the author of several journal publications.

Anita F. Sommer is a Research Associate at the Engineering Design Centre, University of Cambridge, UK. Her research interests are primarily within product development management and engineering design process modelling. Her research focuses on resilient processes that constitute hybrids between standardisation and agile processes in industry. She obtained her PhD degree on product development management in 2014, and her master in industrial engineering in 2009 from Aalborg University, Denmark.

P. John Clarkson is director of the Engineering Design Centre and Professor at the University of Cambridge Engineering Department. His research interests are in the general area of engineering design, particularly the development of design methodologies to address specific design issues, for example, process management, change management, healthcare design and inclusive design. As well as publishing over 450 papers, he has written and edited a number of books on medical equipment design and inclusive design. 